\title{
Suppressing Coherent Noise in Radar Applications With Long Dwell Times
}

\author{
Christopher T. Allen, Senior Member, IEEE, Shadab N. Mozaffar, Student Member, IEEE, and Torry L. Akins
}

\begin{abstract}
A method for suppressing internally produced coherent noise in radar applications is presented and experimentally demonstrated. The technique enhances conventional coherent averaging and involves interpulse phase modulation that is introduced digitally in the transmit waveform and removed digitally following digitization in the receiver. Experimental demonstration of this concept resulted in an additional noise floor reduction of 15 to $20 \mathrm{~dB}$ (compared to the conventional coherent averaging alone) when the number of coherent averages was between 1000 to 100000 , beyond which no significant suppression was observed.
\end{abstract}

Index Terms-Radar signal processing.

\section{INTRODUCTION}

W HILE MOST radar systems operate with limited dwell times due to system geometry dynamics or changing target characteristics, some remote sensing applications offer extraordinarily long dwell times (i.e., the period that the radar observes a particular target). One such example is ice-sheet probing with a slow-moving surface-based $150-\mathrm{MHz}$ synthetic aperture radar (SAR). With a $4-\mathrm{m} / \mathrm{s}$ vehicle speed, a $5-\mathrm{kHz}$ pulse repetition frequency, and a $500-\mathrm{m}$ synthetic aperture length, each pixel will incorporate echoes from more than 600000 pulses received from the ice sheet's basal surface. In such systems, the use of long dwell times allows the radar to operate with a reduced transmit power level without degrading the radar sensitivity by averaging the echo signals to reduce the random noise level. Due to its nonrandom nature, coherent noise is not reduced by averaging, and, although it is typically much smaller than random noise, if present it represents a limit to this dwell-time/transmit power tradeoff.

Radar sensitivity is determined by the ability to reliably detect weak signals in the presence of noise. Key noise sources in radar remote sensing systems include thermal noise, quantization noise, and coherent noise. Various techniques have been developed to reduce the noise without degrading the signal thus improving radar sensitivity. These techniques exploit characteristic differences between the noise and the desired signal. We present a technique for reducing coherent noise without adversely affecting the signal. This technique, when used in conjunction with known techniques for reducing thermal and quantization noise, significantly extend our ability to recover weak radar signals well below the various noise levels.

\footnotetext{
Manuscript received January 7, 2005; revised March, 2, 2005. This work was supported in part by the National Science Foundation under Grant OPP-0122520 and in part by the National Aeronautics and Space Administration under Grant NGT5-30449.

The authors are with the Department of Electrical Engineering and Computer Science, University of Kansas, Lawrence, KS 66045 USA (e-mail: callen@eecs.ku.edu).

Digital Object Identifier 10.1109/LGRS.2005.847931
}

Radar systems whose pulse repetition frequency exceeds the Nyquist sampling rate are said to be oversampled and by oversampling significantly permit the application of various techniques for reducing the received noise power. A technique known as stacking or coherent averaging [1] is effective at reducing thermal noise, which is typically characterized as bandlimited white noise characterized by a zero-mean Gaussian probability density function. This technique is applicable when the radar's target or scene is oversampled so that successive radar echo signals are added together to produce an aggregate echo signal. For example, when the signal of interest is essentially static, averaging $N$ samples yields a constant signal amplitude (and constant signal power). Simultaneously, averaging $N$ uncorrelated noise samples reduces the variance of the noise (proportional to the noise power) resulting in a net signal-to-noise power ratio (SNR) improvement of $N$. (In SAR image-formation processing this concept is extended whereby echo signals from a scatterer viewed over a range of observation angles are coherently combined (via a focusing process) to improve the SNR.) This well-known technique is applied widely and provides significant SNR improvement so long as the assumptions are satisfied.

However, not all noise sources have the same characteristics as thermal noise. For example quantization noise, which is a byproduct of employing finite-resolution analog-to-digital converters (ADCs), is correlated with the signal. Consequently, when the thermal noise is much less than the quantization noise level, the correlated quantization noise will limit the achievable signal detection and stacking will not help. A technique for reducing the quantization noise level known as dithering [2] involves ensuring that uncorrelated noise is digitized along with the desired signal. The presence of uncorrelated noise randomizes the quantization noise so that stacking becomes an effective technique for reducing its effects. Thus in applications where the signal-to-noise ratio is very small ( $\mathrm{SNR}<1$ ), the conditions for dithering are therefore satisfied and the quantization noise effects are reduced [1, p. 17].

Coherent noise not associated with the ADC's quantization noise can also interfere with reliable detection of weak radar signals. While coherent noise could arise from many sources, the radar system itself is often the dominant source. Coherent radar systems typically rely on a master oscillator from which various frequencies and timing signals are derived. Leakage of these signals into the receiver signal path or into the ADC represent coherent noise that is immune to the noise suppression benefits of stacking and dithering. Thus continued application of these noise-reduction techniques beyond the point where the coherent noise is dominant is fruitless unless a technique for suppressing it is applied. 


\section{INTERPULSE $0 / \pi$ MODULATION}

Our objective is to reduce the level of coherent noise in our radar data. To accomplish this we apply interpulse $0 / \pi$ phase modulation, a well-known technique used widely to resolve range and Doppler ambiguities and for removing dc offsets [3]. In this new application, for this technique, rather than transmitting a train of identical pulses, we transmit a series of pulses where the odd-numbered pulses have a $\pi$ phase shift relative to the otherwise identical even-numbered pulses. (Our use of the term pulse includes long-duration chirp waveform pulses.) Once received, the $\pi$ phase shift is removed from the echoes corresponding to the odd-numbered pulses, so that during stacking they add constructively with the even-numbered pulses. The coherent noise produced internally, on the other hand, does not experience the alternating $0 / \pi$ phase shift so that when it is received and it experiences the compensating phase shift of the odd-numbered pulses, it combines destructively with the sample of coherent noise that accompanied the even-numbered pulse. The result is a reduction or cancellation of the coherent noise while preserving the desired signal. Whereas other interpulse phase coding could be applied to produce more complex filtering, simply alternating between 0 and $\pi$ phase shifts is sufficient to demonstrate the benefit of this approach.

While implementation in the analog domain would introduce amplitude and phase imperfections that will limit the cancellation of the coherent noise, nearly perfect implementation can be realized when the introduction and removal of the $\pi$ phase shift occurs in the digital domain. This is realized by digitally generating two transmit pulses differing only in their phase and then removing the phase shift following the ADC (the phase shift removal can be easily accomplished by taking the 1's complement of the digital data, i.e., by exclusive-ORing the digitized signal with a 1 for odd-numbered pulses and 0 for even-numbered pulses).

\section{EXPERIMENTAL SETUP}

To test the proposed concept for coherent noise reduction, laboratory experiments were conducted using our multifrequency monostatic synthetic aperture radar (SAR) system. We configured our SAR to operate at a center frequency of 350 $\mathrm{MHz}$ and a bandwidth of $40 \mathrm{MHz}$. In this configuration, the $\mathrm{SAR}$ receiver has a noise figure of $1.43 \mathrm{~dB}$ and a receiver bandwidth of $40 \mathrm{MHz}$. After the received signal is downconverted to a baseband signal with a center frequency of $30 \mathrm{MHz}$, it is digitized using an Analog Devices' AD 9430 12-bit ADC clocked at a $120-\mathrm{MHz}$ rate. The receiver gain is adjustable to provide a noise power at the ADC input of 3,6 , and $10 \mathrm{~dB}$ above the ADC noise floor of $-64 \mathrm{dBm}$, defined here as the total in-band integrated noise power. (In-band refers to the $10-50-\mathrm{MHz}$ band which is the band of interest for the SAR receiver.) The digitized data are captured in a first-in first-out (FIFO) memory and subsequently averaged $N$ times. In our SAR, all signals (timing and waveform) are derived from a very stable $10-\mathrm{MHz}$ rubidium-based oscillator.

Two configurations were used to conduct the coherent noise reduction tests. In the first configuration, the receiver input was terminated with a 50- $\Omega$ load and no radar signal was injected

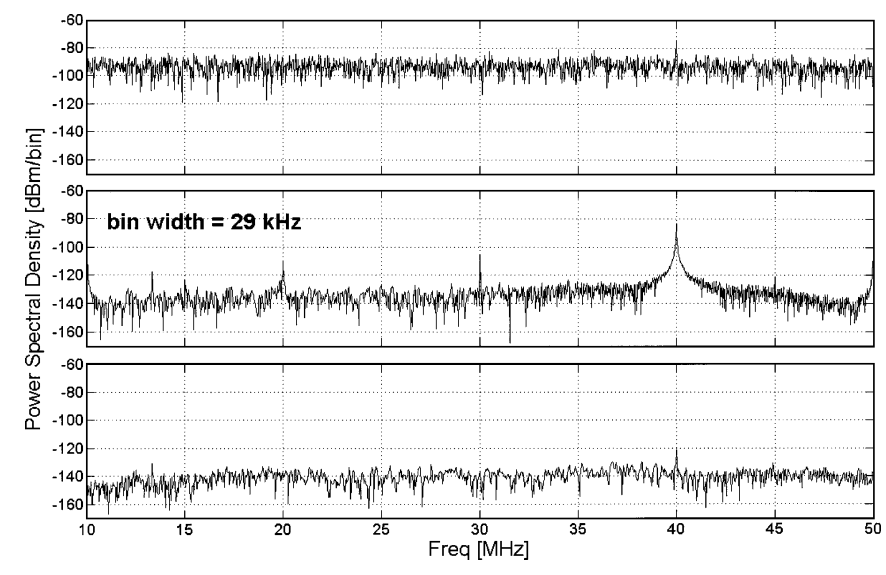

Fig. 1. Measured power spectra of noise digitized at a $120-\mathrm{MHz}$ rate. Top plot is with no averaging. Middle and bottom plots are spectra after one million averages without and with $0 / \pi$ phase modulation, respectively.

into the receiver front-end. In the second, a $0 / \pi$ phase modulated radar signal was injected into the receiver with a signal power below the ADC noise floor.

\section{EXPERIMENTAL RESULTS}

\section{A. Noise Only}

With the receiver input terminated with a $50-\Omega$ load and the receiver gain set to cause the thermal noise to be $3 \mathrm{~dB}$ greater than the ADC noise floor, receiver data were collected and processed both with and without the $0 / \pi$ interpulse modulation. Fig. 1 shows the power spectral density of the processed data. After digitizing about 5000 data points of receiver noise, the power spectral density is found by performing a 4096-point fast Fourier transform (FFT) on the data vector. No window functions were applied. Following the FFT, the magnitudes of the data were converted into decibels.

In the top plot, no averaging was performed and the spectrum associated with white noise is observed, however the $40-\mathrm{MHz}$ spur at around $-74 \mathrm{dBm}$ is well above the $-95 \mathrm{dBm} / \mathrm{bin}$ nominal noise floor. (Note that the bin width is $29 \mathrm{kHz}$ in this dataset.) The total noise power between 10 and $50 \mathrm{MHz}$ is about $-61 \mathrm{dBm}$ which includes the $40-\mathrm{MHz}$ spur.

The middle plot shows a power spectral density of the noise data after averaging one million independent 4096-point vectors. As expected the incoherent noise is largely suppressed while coherent signals can be clearly seen at the system clock frequency and its harmonics $(10,20,30,40$, and $50 \mathrm{MHz})$ as well as other frequencies, with the $40-\mathrm{MHz}$ component being the most dominant at $-83 \mathrm{dBm}$. The reduction of the $40-\mathrm{MHz}$ component may indicate that it is not entirely coherent, and further investigation showed that the amplitude of the $40-\mathrm{MHz}$ signal fluctuates. The suppression of the noise floor level by less than $60 \mathrm{~dB}\left(N=10^{6}\right)$ indicates that a portion of the broadband noise may be coherent due to spectral leakage from the clock and its harmonics. The total noise power between $10-50 \mathrm{MHz}$ is now about $-82 \mathrm{dBm}$.

The bottom plot shows the result when the $0 / \pi$ phase modulation was applied to another one million independent 5000-point vectors of digitized noise. The benefit of the phase modulation concept is evident as the strong coherent terms are significantly 


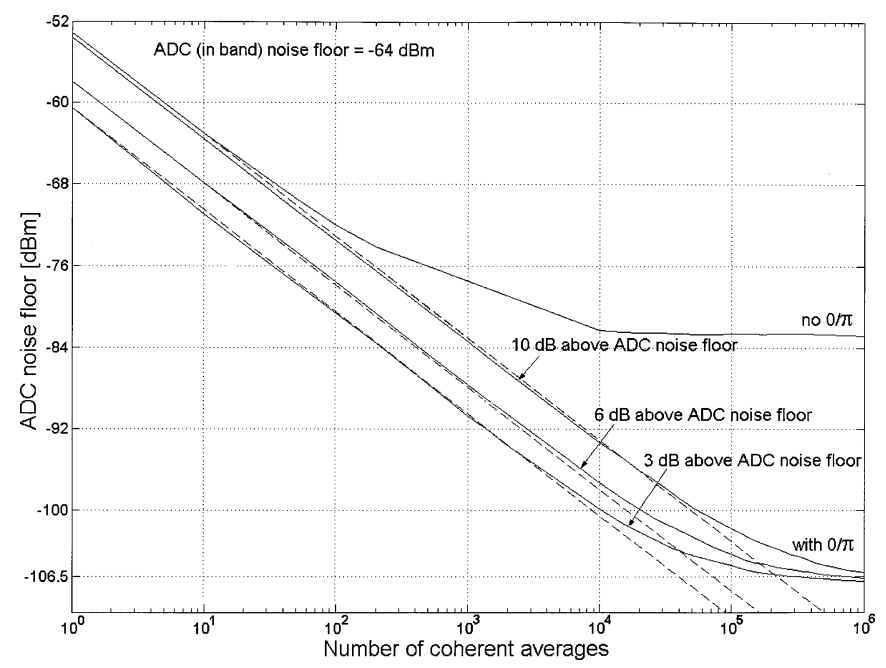

Fig. 2. Measured total ADC noise (in the $10-50-\mathrm{MHz}$ band) as a function of the number of coherent averages.

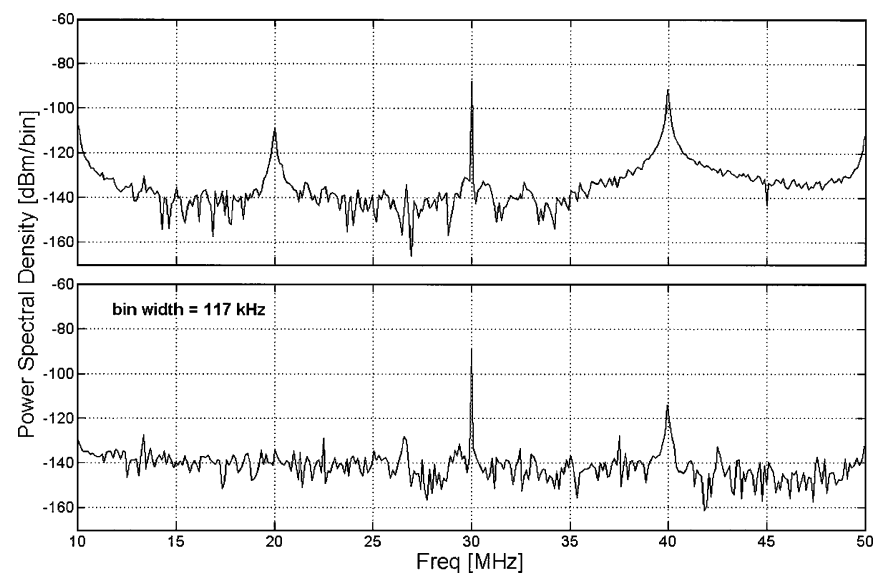

Fig. 3. Measured power spectra of a $30-\mathrm{MHz}$ signal in noise digitized at a $120-\mathrm{MHz}$ rate. Top and bottom plots are spectra after one million averages without and with $0 / \pi$ phase modulation, respectively.

reduced. Whereas without $0 / \pi$ phase modulation the largest frequency spur was $-83 \mathrm{dBm}$, with $0 / \pi$ applied, this component is reduced by almost $40 \mathrm{~dB}$ to $-121 \mathrm{dBm}$. Spurs at $10,20,30$, and $50 \mathrm{MHz}$ are also suppressed to at least the background noise level. Interestingly the spur at $13 \mathrm{MHz}$ persists although reduced by about $13 \mathrm{~dB}$. The $0 / \pi$ modulation also reduces the power of the background noise level (ignoring the spurs) by about $10 \mathrm{~dB}$. Overall the total noise power between $10-50 \mathrm{MHz}$ is about $-106 \mathrm{dBm}$, a reduction of about $24 \mathrm{~dB}$ compared to the no- $0 / \pi$ case.

Using the total noise power as the figure of merit, the benefit of additional coherent averages were investigated. Measured data for cases without and with $0 / \pi$ phase modulation were analyzed with the number of coherent averages varying from one to one million, with the results shown in Fig. 2. Additionally, three different receiver gains were applied producing noise levels at the ADC input that are 3,6, and $10 \mathrm{~dB}$ above the ADC noise floor.

In all cases coherent averaging produces the expected reduction in noise floor up to about 100 averages beyond which the power from the coherent spurious components begin to dominate the noise in the non- $0 / \pi$ case. For this case, the incremental benefit from additional coherent averaging decreases up to 10000 averages beyond which no discernable improvement results. For the cases involving $0 / \pi$ phase modulation, the benefit from additional averaging continues to agree with the theoretical predictions (dashed lines) up to about 10000 averages, beyond which the incremental benefits continue up to about 100000 averages. For all three initial input noise levels (3, 6, and $10 \mathrm{~dB}$ above the $\mathrm{ADC}$ noise floor) the final ADC noise floor after one million averages is the same, about $-106.5 \mathrm{dBm}$. Further averaging produces no significant benefits. Overall, the application of $0 / \pi$ phase modulation provides about $25 \mathrm{~dB}$ of additional ADC noise floor suppression by reducing the spurious noise power.

\section{B. Weak Signal}

In the second experimental setup, a coherent time-gated $30-\mathrm{MHz}$ sinusoid with a $10-\mu \mathrm{s}$ duration and a $-88 \mathrm{dBm}$ signal power was injected into the receiver front end. At this power level, the signal-to-noise ratio at the ADC input is less than $-28 \mathrm{~dB}$. The signal and noise are digitized at a $120-\mathrm{MHz}$ rate producing a 1200-point data vector. Using a 1024-point FFT, the power spectral density is obtained which is then converted into decibels. Again, only frequencies between $10-50 \mathrm{MHz}$ are presented. (Note the bin width is now $117 \mathrm{kHz}$.)

The power spectral density obtained after one million conventional coherent averages is shown in the top plot of Fig. 3. In addition to the $30-\mathrm{MHz}$ signal, spurs at $10,20,40$, and $50 \mathrm{MHz}$ are also well above the noise floor power, $40 \mathrm{MHz}$ being the dominant spur with a power of about $-91 \mathrm{dBm}$.

The bottom plot in Fig. 3 shows the results when $0 / \pi$ phase modulation was applied. After one million averages, the $30-\mathrm{MHz}$ signal remains at full strength whereas the spurious signals are significantly reduced. The previously dominant 40-MHz spur remains above the noise floor; however, its power level is now $-114 \mathrm{dBm}, 23 \mathrm{~dB}$ lower than in the case without $0 / \pi$ phase modulation. The overall noise floor remains at about $-140 \mathrm{dBm} / \mathrm{bin}$.

\section{CONCLUSION}

The use of interpulse $0 / \pi$ phase modulation with coherent averaging has been experimentally demonstrated to significantly suppress coherent noise. In our demonstration this technique resulted in an additional noise floor reduction of 15 to $20 \mathrm{~dB}$ (compared to coherent averaging alone) when the number of coherent averages was between 1000 to 100000 , beyond which no significant suppression was observed. Clearly integrating 1000 to 100000 pulses requires long observation times that may be found in long-dwell pulsed radar systems or in SAR systems wherein long synthetic apertures are involved. Both the signal synthesis and data analysis components of this technique were implemented digitally with no additional hardware required and negligible impact on processing requirements.

\section{REFERENCES}

[1] B. C. Brock, "The role of noise in analog-to-digital converters," Sandia Nat. Lab., Sandia, NM, SAND Rep. SAND96-2697, Nov. 1996.

[2] M. Bartz, "Large-scale dithering enhances ADC dynamic range," Microwaves RF, vol. 32, pp. 192-198, May 1993.

[3] B. G. Laird, "On ambiguity resolution by random phase processing," in Proc. 20th Conf. on Radar Meteorology, Boston, MA, 1981, pp. $327-331$. 